\title{
Unique genetic and environmental determinants of prolonged fatigue: a twin study
}

\author{
I. HICKIE, ${ }^{1}$ K. KIRK AND N. MARTIN \\ From the School of Psychiatry, University of New South Wales and Academic Department of Psychiatry, \\ St. George Hospital and Community Health Service, Sydney, NSW; and Queensland Institute of Medical \\ Research, Brisbane, Queensland, Australia
}

\begin{abstract}
Background. Prolonged fatigue syndromes have been proposed as prevalent and disabling forms of distress that occur independently of conventional notions of anxiety and depression.

Methods. To investigate the genetic and environmental antecedents of common forms of psychological and somatic distress, we measured fatigue, anxiety, depression and psychological distress in 1004 normal adult twin pairs (533 monozygotic (MZ), 471 dizygotic (DZ)) over 50 years of age.

Results. Familial aggregation of psychological distress, anxiety and fatigue appeared to be due largely to additive genetic factors (MZ:DZ ratios of 2.12-2.69). The phenotypic correlations between the psychological measures (distress, anxiety and depression) were moderate $(0 \cdot 67-0 \cdot 79)$ and higher than that between fatigue and psychological distress $(0 \cdot 38)$. Multivariate genetic modelling revealed a common genetic factor contributing to the development of all the observed phenotypes (though most strongly for the psychological forms), a second independent genetic factor also influenced anxiety and depression and a third independent genetic factor made a major contribution to fatigue alone. In total, $44 \%(95 \%$ CI $25-60 \%)$ of the genetic variance for fatigue was not shared by the other forms of distress. Similarly, the environmental factor determining psychological distress made negligible contributions to fatigue, which was underpinned largely by its own independent environmental factor.
\end{abstract}

Conclusion. This study supports the aetiological independence of prolonged fatigue and, therefore, argues strongly for its inclusion in classification systems in psychiatry.

\section{INTRODUCTION}

Prolonged fatigue constitutes a common form of distress in the community, and is particularly prevalent $(10 \%$ to $25 \%)$ in primary care settings (Chen, 1986; David et al. 1990; Angst \& Koch, 1991; Kirmayer \& Robbins, 1991; Bates et al. 1993; Pawlikowska et al. 1994; Üstün \& Sartorius, 1995; Hickie et al. 1996; Mason \& Wilkinson, 1996; Wessely et al. 1996). These syndromes are characterized by disabling mental and physical fatigue, which is not relieved by rest, and associated neuropsychological and

${ }^{1}$ Address for correspondence: Professor Ian Hickie, Academic Department of Psychiatry, St. George Hospital and Community Health Service, 7 Chapel Street, Kogarah, NSW 2217, Australia. neuromuscular symptoms (Hickie et al. 1997). When severe and prolonged then specific syndromal disorders such as chronic fatigue syndrome (CFS) (duration $>6$ months; Fukuda et al. 1994) and 'neurasthenia' (duration $>3$ months; ICD-10, World Health Organization, 1992) are diagnosed. The WHO multicentre study of primary care (Üstün \& Sartorius, 1995) demonstrated that neurasthenia was indeed prevalent across all sites (mean $5.4 \%$, range $1 \cdot 1-10 \cdot 5 \%$ ), while CFS appears to be present in $0.5-2.5 \%$ of patients attending general practitioners (Hickie et al. 1996; Wessely et al. 1996).

By contrast, other international psychiatric classification systems, notably DSM-IV (American Psychiatric Association, 1994) and statistical 
models of the common forms of psychological distress (Goldberg et al. 1987; Goldberg \& Bridges, 1991; Goldberg, 1996) have tended to discount the notion of separate fatigue syndromes. Within these systems, fatigue states are assumed to represent largely the somatic symptoms of more typical (or unrecognized) anxiety and/or depressive states. While fatigue states are frequently co-morbid with anxiety and depressive disorders, between a third and a half of patients with CFS do not meet lifetime criteria for any other psychiatric disorder (Kroenke et al. 1988; Wessely \& Powell, 1989; Hickie et al. 1990) and, similarly, a third of patients with prolonged fatigue in primary care do not meet criteria for other psychological disorders (Hickie et al. 1996).

Patients with CFS do not show a specific response to antidepressant pharmacotherapy (Wilson et al. 1994; Vercoulen et al. 1996) and longitudinal data sets confirm that patients with prolonged fatigue/neurasthenia tend to maintain their unique characteristics over time (Merikangas \& Angst, 1994; Hickie et al. 1998 a). Therefore, on the basis of epidemiological, longitudinal and treatment data it has been argued (see Hickie et al. 1997, 1998 b) that such fatigue syndromes are worthy of specific investigation. A most important test of the nosological validity of prolonged fatigue states, however, is whether such syndromes are aetiologically independent of anxiety and depression.

Twin studies provide a unique opportunity to test various aetiological models of the common mental disorders (Kendler et al. 1986, 1987). By using subjects who are not attending healthcare practitioners, not only do population-based twin studies provide aetiological insights but the results are not confounded by other potential risk factors to healthcare-seeking behaviour. To date, this method has been used largely to study the complex genetic and environmental determinants of the common psychological phenotypes of anxiety and depression. While such studies support the role of additive genetic factors in determining the observed familial aggregation of psychological distress, multivariate modelling has suggested that anxiety and depression are largely underpinned by one genetic factor, with independent environmental factors most likely giving rise to the differences in observed phenotypes (Kendler et al. 1986,
1987, 1992; Roy et al. 1995; Kendler, 1996). In fact, recent data suggest that genetic factors predispose the individual to experiencing certain environmental stressors (Kendler \& KarkowskiShuman, 1997).

Although familial aggregation has been suggested for clinic-based cases of CFS, this does not determine whether such aggregation is due to genetic and/or environmental factors. In another study where we used the twin model to study the complex relationships between psychological distress, prolonged fatigue and immune function (Hickie et al. 1999), we demonstrated that additive genetic factors accounted largely for familial aggregation of psychological distress and prolonged fatigue (but not immune function), and, most importantly, that prolonged fatigue appeared to have unique genetic and environmental determinants. However, the study was limited by the small sample size and, hence, may have underestimated the size of other important effects.

In this study, our aims were to: (i) investigate the genetic and environmental determinants of the common phenotypes of distress by including not only conventional measures of anxiety, depression and psychological distress but also a relevant measure of prolonged fatigue; and, (ii) to determine whether our earlier model could be replicated in a much larger twin sample. To these ends, we have measured these various forms of psychological distress and fatigue in an unselected sample of 1004 twin pairs (533 monozygotic (MZ), 471 dizygotic (DZ)) aged $>50$ years. Conventional modelling would predict that prolonged fatigue would not be associated with any specific genetic or environmental determinants, while our hypothesis suggests that such syndromes are not only strongly heritable but that they have unique genetic and/or environmental determinants.

\section{METHOD}

Data for this study were collected in the context of a Health and Lifestyle Questionnaire of twins enrolled on the Australian NHMRC Twin Registry who were aged $>50$ years (the 'Over 50s Twin Study'). A 16-page questionnaire was mailed during 1993-5 to 2281 pairs of eligible twins, with the mailing undertaken in three waves in order to avoid overburdening twins 
already participating in other studies. Follow-up procedures included the mailing of two separate reminder notices to individuals who had not returned questionnaires, and the use of an abbreviated two-page telephone survey with those who refused to complete the full questionnaire.

Included in the self-report questionnaire was an 8-item version of the Schedule of Fatigue and Anergia (SOFA) - Community version), a screening test for chronic fatigue/neurasthenia (Hickie et al. 1996), the 12-item General Health Questionnaire (GHQ) (Goldberg \& Williams, 1988), a screening test for psychiatric morbidity, and the anxiety and depression scales of the Delusion Symptoms States Inventory (DSSI/ sAD - Bedford et al. 1976). The 10-item SOFA was developed to identify cases of severe and disabling fatigue of at least 1 month's duration. When this instrument was adapted for use in primary-care settings, it was found that $25 \%$ of patients reported three or more positive responses and were classified as cases of prolonged fatigue. In our other twin study (Hickie et al. 1999), this cut-off score identified $9 \cdot 2 \%$ of the sample as SOFA cases. In this study, we reduced the number of items from 10 to eight, omitting two items which directly overlapped with comparable GHQ items ('sleeping poorly' and 'poor concentration'), gave the general instruction for duration as 'over the past few weeks' and simplified the responses to 'yes' or 'no'. Responses were then summed to give a total score for fatigue and related somatic symptoms (FAT: range $0-8)$.

The 12-item GHQ has been used extensively in epidemiological studies to detect cases of psychological distress (DIS). The instrument is designed principally to detect those common forms of anxiety and depression which dominate presentations in primary care (Goldberg \& Williams, 1988). Modelling of responses suggests that GHQ responses can be explained either by one general dimension of distress or two moderately-correlated dimensions of anxiety and depression (Goldberg et al. 1987; Goldberg, 1996). A variety of scoring systems have been proposed for the four-point severity scale, with the suggestion that more chronic symptoms may be underestimated by traditional approaches to scoring (Goodchild \& Duncan-Jones, 1985). Therefore, proposed GHQ scoring systems were
considered-Goldberg \& Williams' standard scoring system (1988), a Likert scoring method, and a chronicity scoring method (Goodchild \& Duncan-Jones, 1985). Polychoric correlations between the various GHQ scoring methods and the DSSI/sAD anxiety and depression and SOFA scores demonstrated only marginal differences between the results for the standard and chronicity scoring methods, although these had somewhat higher correlations than those obtained using Likert scoring. Consequently, the standard GHQ scoring was used in those analyses. Each item is scored dichotomously (0-0-1-1: following the sequence of response categories) and item scores are then summed for a total score (DIS: range 0-12).

In the DSSI/sAD the anxiety (ANX) and depression (DEP) scales each comprise 7 items (scored 0-3) giving total scores ranging from 0-21 for each scale. These scales have been used extensively in previous twin studies examining the genetic and environmental determinants of anxiety and depression. By contrast with the GHQ, reported depression scores are generally of lower prevalence and greater severity, suggesting cases more akin to anxiety and depression seen in clinical settings.

\section{Zygosity diagnosis}

Zygosity of twins was decided on the basis of their responses to standard questions about similarity and the degree to which others confused them. Pairs giving inconsistent responses were recontacted for clarification. Such procedures have been shown to given at least $95 \%$ agreement with diagnosis based on extensive blood-typing (Martin \& Martin, 1975; Ooki et al. 1990). More recently, a subsample of 198 same-sex pairs who reported themselves to be $\mathrm{MZ}$ were typed for 11 independent highly polymorphic markers in the course of an asthma study, with no errors in previous zygosity diagnosis detected (Duffy, 1994).

\section{Statistical methods}

Data were analysed using PRELIS 2.12e and LISREL 8.12e (Joreskog \& Sorbom, 1993). The imputation option of PRELIS 2.12e was used to impute missing items responses for the SOFA, GHQ and DSSI scales, within individuals by sex. This approach obtains the substitute value from other cases with similar response patterns 
but no missing values, provided the variance for these other cases is acceptable. Ninety-five item responses were imputed, representing only $0.1 \%$ of all item responses, but increasing the number of individuals with 'complete' data from 2623 to 2703. Listwise deletion, however, had a drastic effect on the number of twin pairs available for study but imputation increased the total number of pairs available from 946 to 1004 .

Correlations between variables are calculated on the assumption each variable represents a continuum of liability which is normally distributed in the population. In the case of the psychological variables, which have a finite number of categories (13 for DIS, 9 for FAT, and 22 each for ANX and DEP) and skewed distributions (most people with low scores and a long tail to the right), it is most convenient to treat them as categorical (grouping rare responses to the right hand side of the distributions) and calculate polychoric correlations using PRELIS. This is necessary to avoid sparse contingency tables with lots of empty cells which prevent estimation of the polychoric correlation. Thus, FAT was reduced to a 6-point scale, ANX to a 5-point scale, and DIS and DEP to 4-point scales.

Significant twin correlations establish the fact that there is familial aggregation for the measures of interest. Our task, however, is to distinguish between the possible mechanisms by which this familial likeness may arise. The accepted method is via structural equation modelling as implemented in LISREL, Mx, or similar packages (Neale \& Cardon, 1992). One can conceive of three broad causes of variation, two of which (additive genetic influences ' $G$ ' and common environment ' $C$ ') make family members more alike than random pairs of individuals, and one of which (unique environmental experiences ' $E$ ') makes MZ twins and siblings different. The task then is to decide which combination of these three parameters provides the most parsimonious explanation for the observed pattern of $\mathrm{MZ}$ and $\mathrm{DZ}$ twin correlations.

The methods of structural equation modelling are readily extended to the more complex questions of the relationship between variables, in which one is trying to discover not only the sources of covariation (G, C, E), but the pattern or structure in which these differentially influence the covarying measures. Specifically, we wish to know whether there are unique genetic determinants of fatigue which do not influence our other measures of affect - anxiety, depression and psychological distress. We have therefore, used the Cholesky decomposition to dissect this (Neale \& Cardon, 1992). Each source of covariance between $n$ variables is decomposed into a series of $n$ factors, the first factor loading on all variables, the second factor loading on all but the first variable, the third factor loading on all but the first two variables, and so on until the last factor loads on only the $n$th variable. It is this last factor that provides the estimate of unique variance, and for this reason we enter the fatigue measure last in our analysis. Other factor models, such as the independent pathways model and the common pathway model (Neale \& Cardon, 1992) can be fitted to the data to perhaps provide more economical descriptions of the covariation between variables, but this is not our primary purpose here.

Models were fitted in LISREL 8.12 using the weighted least-squares fit function which employs the asymptotic covariance matrices estimated by PRELIS. We began by specifying a complete decomposition for three sources of variance - additive genes, shared environment, and individual environment. This full model was then simplified by successive dropping of nonsignificant parameters i.e. by seeing whether dropping a parameter resulted in a significant increase in the goodness-of-fit chi-square. The same principles of parsimony were applied in arriving at the preferred model (Neale \& Cardon, 1992).

\section{RESULTS}

Full questionnaire responses were received from 1279 complete pairs and 558 singles, and a further 286 individuals participated in a telephone interview. The individual and complete pairs response rates (excluding deaths and noncontacts) were $71 \%$ and $61 \%$ respectively. Individual response rate differed between females and males $(74 \%$ v. $60 \%), \mathrm{MZ}$ and $\mathrm{DZ}$ twins $(74 \%$ v. $65 \%)$, and was greater in the middle of the age range (75\% in the 70-74 year olds) than at either the lower or upper extremes $(67 \%$ in $50-54$ year-olds and $61 \%$ in those $>85)$. 
After imputation as described above, complete results for SOFA, GHQ and DSSI were available for 2703 individuals ( $59 \%$ of those mailed) and 1004 complete pairs (44\% pairwise response). Mean age of the sample was 61.9 years, but this was highly skewed, with $49 \%$ aged $50-59,32 \%$ aged $60-69,15 \%$ aged $70-79$ and only $4 \%$ aged $\geqslant 80$. The sample was predominantly female $(69 \%)$.

Of the 1004 pairs in our present sample, 37 pairs $(3.7 \%)$ had also taken part in a parallel study of psychological effects on immune functioning (Hickie et al. 1999). This study also employed the GHQ and the full version of the SOFA. The mean inter-test period was 269 days (range 44-464) and the test-retest correlations for GHQ and SOFA scores in these 74 individuals were 0.33 and 0.43 respectively. Interestingly, these are almost identical to the corresponding MZ twin correlations for DIS and FAT of 0.35 and 0.43 (see below), suggesting that an individual is as similar to his/her MZ co-twin as him/herself over time.

Of the total sample $(N=2703) 9 \%$ were categorized as fatigue cases $(\mathrm{FAT}=\mathrm{SOFA}$ scores of five or more) and $13 \%$ described current psychological distress $(\mathrm{DIS}=\mathrm{GHQ}$ scores of three or more), giving rates comparable with our earlier twin study. As expected, 14\% and $7 \%$ of cases were categorized as anxious (ANX) or depressed (DEP) respectively by DSSI criteria. In total, $25 \%$ of the subjects were a 'case' of DIS, FAT, ANX or DEP. No significant sex differences in means were observed for the SOFA and GHQ measures (Wilcoxon two-sample test), but differences between males and females were observed for the anxiety $(P<0.0001)$ and depression $(P<0.001)$, with females having higher scores. Principal component analysis of the responses to the questionnaire items from the SOFA, GHQ and DSSI/sAD confirmed the independence of the various measures.

Comparison of the means of pairs who both completed the questionnaire with those pairs where only one responded can indicate response bias (Neale \& Eaves, 1993). For all four measures the means of complete versus incomplete male pairs were not significantly different. In female twins, there were no differences between complete pairs and single twins for our target variable FAT, or for DIS, but single female twins did have higher anxiety $(P=0.005)$ and depression $(P=0 \cdot 0002)$ scores than complete female pairs indicating that more anxious and depressed pairs may have been less inclined to participate in the study.

\section{Estimating twin correlations}

Complete data were available for 1004 twin pairs, comprising $533 \mathrm{MZ}$ pairs (400 female and 133 male), and $471 \mathrm{DZ}$ pairs (207 female, 67 male and 197 unlike sex pairs). Since some of these group sizes are at the lower limit for calculating polychoric correlations it is desirable to pool the data across sexes into total MZ versus total DZ groups. A concern in pooling over sexes within zygosity groups is that sex differences in means will inflate twin correlations and distort any factor structure. For this reason, we have run multivariate analyses on male and female data separately, as well as the pooled MZ and DZ correlations. Age is always a potential confounding factor, but in this restricted age sample we found only negligible correlations with DIS, FAT, ANX and DEP, none accounting for more than $1 \%$ of variance.

Cross-twin-cross-trait polychoric correlations were estimated using PRELIS for each sexzygosity group and also for the pooled MZ (533 pairs) and DZ (471 pairs) groups. The latter are shown in Table 1 for both MZ and DZ twins. The twin1-twin2 correlations for the four key variables are highlighted in bold. For GHQ, ANX and FAT the MZ correlations are two or more times their DZ counterparts $(0.35$ v. 0.13 , 0.36 v. 0.17 and 0.43 v. 0.16 respectively), indicating genetic control of familial aggregation for these measures.

On the other hand, MZ and DZ correlations for DEP are approximately equal (0.28 v. 0.26), indicating that shared family environment may play a role in familial resemblance for this variable. However, it must be noted that information about the genetics of DEP lies not only in these two correlations, but also in all the other cross-twin-cross-trait correlations involving DEP seen in Table 1. Inspection of these for MZ and DZ twins suggests that DEP may be more genetic than it appears to be from the trait correlations alone - a finding borne out by the results of multivariate genetic analysis.

This is confirmed by preliminary univariate model fitting in which a model postulating 
Table 1. Polychoric correlations for $M Z$ (above diagonal) and $D Z$ (below diagonal) pairs for measures of fatigue (FAT), psychological distress (DIS), anxiety $(A N X)$ and depression (DEP) for twin 1 and twin 2. Co-twin correlations for each measure are shown in bold

\begin{tabular}{|c|c|c|c|c|c|c|c|c|}
\hline & \multicolumn{8}{|c|}{ Monozygotic (533 pairs) } \\
\hline & \multicolumn{4}{|c|}{ Twin 1} & \multicolumn{4}{|c|}{ Twin 2} \\
\hline & FAT1 & DIS1 & ANX1 & DEP1 & FAT2 & DIS2 & ANX2 & DEP2 \\
\hline \multicolumn{9}{|l|}{ Twin 1} \\
\hline FAT1 & 1.00 & $0 \cdot 35$ & $0 \cdot 42$ & $0 \cdot 34$ & $0 \cdot 43$ & $0 \cdot 24$ & $0 \cdot 26$ & $0 \cdot 23$ \\
\hline DIS1 & $0 \cdot 45$ & 1.00 & $0 \cdot 70$ & 0.77 & $0 \cdot 27$ & $0 \cdot 35$ & $0 \cdot 32$ & $0 \cdot 29$ \\
\hline ANX1 & $0 \cdot 49$ & $0 \cdot 60$ & 1.00 & $0 \cdot 78$ & $0 \cdot 29$ & $0 \cdot 26$ & $0 \cdot 36$ & $0 \cdot 28$ \\
\hline DEP1 & $0 \cdot 45$ & $0 \cdot 75$ & 0.76 & $1 \cdot 00$ & $0 \cdot 29$ & $0 \cdot 23$ & 0.39 & $0 \cdot 27$ \\
\hline \multicolumn{9}{|l|}{ Twin 2} \\
\hline FAT2 & $0 \cdot 16$ & $0 \cdot 12$ & $0 \cdot 11$ & $0 \cdot 21$ & 1.00 & $0 \cdot 38$ & $0 \cdot 47$ & $0 \cdot 35$ \\
\hline DIS2 & 0.06 & $0 \cdot 13$ & $0 \cdot 15$ & $0 \cdot 20$ & $0 \cdot 38$ & $1 \cdot 00$ & $0 \cdot 72$ & 0.68 \\
\hline ANX2 & $0 \cdot 07$ & $0 \cdot 14$ & $\mathbf{0} \cdot 17$ & $0 \cdot 21$ & $0 \cdot 50$ & $0 \cdot 68$ & $1 \cdot 00$ & $0 \cdot 81$ \\
\hline \multirow[t]{2}{*}{ DEP2 } & $0 \cdot 16$ & $0 \cdot 16$ & $0 \cdot 22$ & $0 \cdot 26$ & $0 \cdot 38$ & $0 \cdot 72$ & $0 \cdot 79$ & $1 \cdot 00$ \\
\hline & \multicolumn{8}{|c|}{ Dizygotic (471 pairs) } \\
\hline
\end{tabular}

shared environment (CE) as the sole source of familial resemblance is rejected for FAT $\left(\chi^{2}{ }_{1}=14 \cdot 05\right), \quad$ ANX $\quad\left(\chi_{1}^{2}=7 \cdot 62\right)$ and DIS $\left(\chi^{2}{ }_{1}=5 \cdot 34\right)$, while a simple genetic model (AE) gives an excellent fit in each case with $41 \%$, $36 \%$ and $34 \%$ of the variance respectively due to additive genetic factors. For DEP, the CE model could not be rejected $\left(\chi^{2}{ }_{1}=0.02\right)$ but the AE model was also compatible with the data $\left(\chi^{2}{ }_{1}=2 \cdot 50\right)$ and gave a heritability of $31 \%$.

\section{Fitting multivariate genetic and environmental models}

Our task, however, is to explore not only the causes of individual differences for each variable but also the causes of covariation between them. Here, the focus of our interest is covariation between various forms of psychological distress and fatigue. In our sample, the phenotypic correlation between distress and fatigue is only modest $(0 \cdot 38)$, consistent with our notion that these syndromes represent somewhat different types of distress (Hickie et al. 1997). The phenotypic correlations between the other measures of psychological distress ranged between 0.67 and 0.79 .

It is only by multivariate genetic analysis (Neale \& Cardon, 1992) that we can estimate the relative contributions of genes and environment to covariation between measures. We have, therefore, used the Cholesky decomposition to dissect these contributions (Neale \& Cardon, 1992). The models are fitted in LISREL 8.12 using weighted least squares. We begin by specifying a complete decomposition for three sources of variance-additive genes, shared environment, and individual environment (ACE model) and this gives a fit of $\chi_{30}^{2}=41.29$. Dropping the shared environment (AE model) only causes an increase in $x^{2}$ of 1.04 for 10 degrees of freedom, so clearly we can dispense with further consideration of the shared environment. However, dropping genetic factors from the ACE model causes an increase in $\chi^{2}{ }_{10}$ of $22 \cdot 18$, showing that additive genes are an important source of variation and covariation between measures. We now concentrate on simplification of the additive genetic factor structure and by successive dropping of nonsignificant parameters (tested by likelihood ratio chi-square test-that is, by seeing whether dropping a parameter results in a significant increase in the goodness-of-fit chi-square) we arrive at the model shown in Fig. 1, which has a goodness of fit $\chi^{2}{ }_{42}=43 \cdot 61$. Only the significant $($ alpha $=0.05)$ path coefficients estimated are shown.

The principal genetic features of this model are as follows. First, there is a common genetic factor $(\mathrm{G} 1)$ accounting for $36 \%\left(0 \cdot 60^{2}\right)$ of the variance in DIS which also accounts for $19 \%$ of the variance in FAT, as well as $23 \%$ and $25 \%$ respectively of the variance in ANX and DEP. A second, independent genetic factor (G2) accounts for $11 \%$ and $9 \%$ of variance in ANX and DEP, and also accounts for $5 \%$ of the 


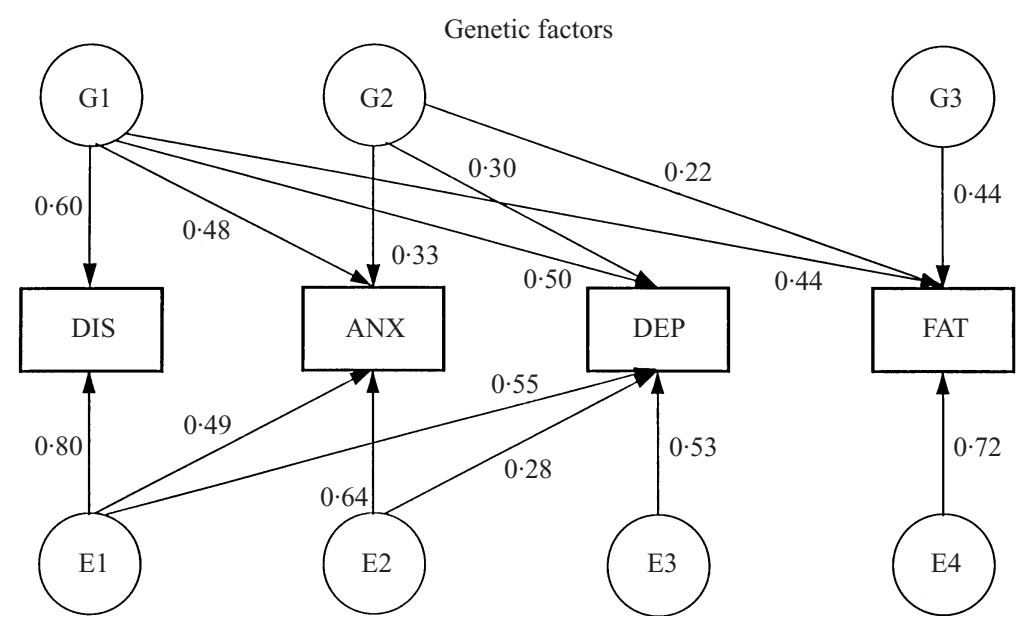

Environmental factors

FIG. 1. Path diagram showing latent genetic and environmental influences (shown in circles) on the measured phenotypes DIS, ANX, DEP and FAT (shown in squares). G1, G2 and G3 represent additive genetic factors and E1, E2, E3 and E4 are unique environmental factors. Numbers by paths are path coefficients and must be squared to obtain proportions of variance of the measured variable accounted for by the latent variable. Paths contributing less than $5 \%$ of the variance are not shown.

variance in FAT. Finally, and most importantly, there is also specific genetic variance for FAT (G3), accounting for $19 \%\left(=0 \cdot 44^{2}, 95 \% \mathrm{CI}\right.$ $10-27 \%)$ of its variance. Thus, of the genetic variance for FAT, $44 \%(19 /(19+5+19), 95 \%$ CI $25-60 \%$ ) is specific to fatigue and not shared with other measures of psychological distress.

We can reorder the variables and refit the Cholesky decomposition to estimate the unique genetic variance for the other three variables. By contrast with fatigue, only $24 \%$ of the genetic variance in DIS is unique, and for ANX and DEP, all genetic factors influencing one also influence the other, replicating earlier findings (Kendler et al. 1987).

Individual environmental variance also subsumes any errors of measurement and so it is to be expected that the vertical paths from E1, E2, $\mathrm{E} 3$ and E4 to the corresponding first, second, third and fourth variables respectively (DIS, ANX, DEP, FAT) will be large. However, while there is some cross loading of the E1 factor on ANX and DEP, accounting for $24 \%(17-33 \%)$ and $30 \%(21-40 \%)$ of variance respectively, the crossloading of the first three environmental factors on FAT is negligible (less than $6 \%$ $(3-9 \%)$ in total). Therefore, as well as being to a large extent genetically distinct from the other distress variables, FAT is also almost completely distinct in terms of its environmental aetiology.
The results presented are for the pooled MZ and DZ twin samples, but separate analyses of the female and male data gave very similar results, demonstrating a high degree of congruence between the sexes. The only exception was that for neither sex was the second genetic factor G2 necessary (presumably because of the reduced power with smaller cell sizes), and this had the effect of making FAT appear even more genetically distinct from the other three variables than in the pooled analysis.

\section{DISCUSSION}

Our major aim in this study was to test whether the common forms of somatic and psychological distress were determined by independent genetic and/or environmental factors. While epidemiological, longitudinal and treatment response data have been used to argue for the validity of prolonged fatigue states (Merikangas \& Angst, 1994; Hickie et al. 1996, 1998a, b; Vercoulen et al. 1996), this study replicates and extends our other much smaller twin study (Hickie et al. 1999) which proposed unique aetiological factors for prolonged fatigue. This study again provides evidence for: $(i)$ the familial aggregation of fatigue being determined largely by additive genetic factors $\left(r_{\mathrm{Mz}}=0.43 \pm 0.05\right.$, $r_{\mathrm{DZ}}=0 \cdot 16 \pm 0 \cdot 05$ ); and (ii) the presence of dis- 
crete genetic and environmental determinants of fatigue, which are not shared by the other measures of psychological distress. There was no evidence for a role for shared family environment in the aetiology of fatigue.

The application of the twin method provides novel insights into the aetiological relationships between somatic and psychological forms of distress. The key features of the final multivariate model developed here (see Fig. 1) are: (i) a common genetic factor (G1) underpins the expression of all forms of distress, but has its most significant effects on psychological rather than somatic symptoms; (ii) a second independent genetic factor (G2) contributes largely to the more severe forms of anxiety and depression; (iii) a third, specific independent genetic factor (G3) contributes to fatigue only; (iv) a unique environmental common factor (E1) is associated with all forms of psychological distress, but not fatigue; and, (v) independent environmental factors (E2-E4) then contribute to the development of each of the other forms of distress measured, including fatigue.

Co-morbidity and longitudinal data from community and clinical settings support the notion that the common forms of anxiety and depression are underpinned by common vulnerability factors (Kessler et al. 1994; Merikangas \& Angst, 1994; Hunt \& Andrews, 1995; Andrews, 1996). Previous twin data suggest largely that one common genetic factor, which acts in combination with differing environmental factors, determines the clinical phenotypes of anxiety and depression (Kendler et al. 1987). The results of this study largely confirm the notion that these common forms of psychological distress do share a common genetic element (G1) but also have some differing environmental determinants (E1-E3). While the large common genetic factor (G1), which underpins psychological distress here also contributes to the development of fatigue, $44 \%$ of the genetic contribution to fatigue was not shared with the other common psychological forms of distress (G3). Furthermore, environmental factors which determined the psychological forms of distress (E1-E3) were almost totally independent of the environmental determinant of fatigue (E4).

In summary, this study suggests that prolonged fatigue states are aetiologically distinct from the other common presentations of psychological distress. This interpretation is consistent with data derived from longitudinal analyses of these syndromes in primary-care settings (Hickie et al. 1998a) where prolonged fatigue does not constitute a risk factor to the development of psychological distress over time, and vice versa. These two types of analyses provide data with regard to aetiological risks that cannot be derived from cross-sectional studies. As such, they are critical steps in establishing the validity of any proposed psychiatric syndrome (Kendell, 1989). Further, as the twin data was collected from persons not attending for medical assessment, the risk factors described are for the phenotype itself and are not confounded by healthcare seeking behaviour (with the latter being intrinsic to risk factor studies based in medical care settings).

There are a number of limitations to this study. First, studies which rely on self-report data will not necessarily converge with interviewbased data. Neurasthenia diagnosed by formal interview in community or medical settings (Üstün \& Sartorius, 1995; World Health Organization, 1992) may prove to have some distinct features. Secondly, this style of twin study may not produce a representative community sample due to a range of sampling biases (particularly, as the analyses require complete data sets from both twins). We have detected evidence that we have undersampled more depressed and anxious female (but not male) twin pairs. However, since our target variable is fatigue, and this is only moderately correlated with DEP and ANX (about $0 \cdot 4$ ), this sampling bias is unlikely to have serious repercussions on our findings. Thirdly, prolonged fatigue in non-medical settings may be aetiologically distinct from the more severe and disabling disorders encountered in specialty medical and psychiatric practice (Fukuda et al. 1994; Hickie et al. 1995). Fourthly, the low prevalence of the more severe anxiety and depressive disorders in this sample limits the interpretations that can be drawn with regard to the genetic and environmental determinants of those disorders.

Neurasthenia and other prolonged fatigue states are now the focus of renewed interest in psychiatric epidemiology and classification (Hickie et al. 1997, 1998b). The concept was appropriately criticized (Wessely, 1990; Gold- 
berg \& Bridges, 1991; Goldberg, 1996) prior to the presentation of sufficient aetiological and treatment data to support its validity. This aetiological study, in combination with the epidemiological, co-morbidity, longitudinal and treatment data now available (see Hickie et al. 1997, 1998 b), provides a strong argument for the inclusion of prolonged fatigue states in future psychiatric classification systems.

Twins from this study are drawn from the Australian NHMRC Twin Registry. This work was partly supported by a donation from Mr George Landers, and benefited from funding from NHMRC to I. B. H. (931215-Project Grant, and 953208-Program Grant) and N.G.M. (941177).

We thank Fran Boyle and Len Roberts for their work in constructing the questionnaire, Olivia Zheng for administering the mail-out, John Pearson for data management and Nirmala Pandeya for data cleaning. Most of all we should like to thank the twins for their cooperation.

\section{REFERENCES}

American Psychiatric Association (1994). Diagnostic and Statistical Manual of Mental Disorders (4th edn). American Psychiatric Association: Washington, DC.

Andrews, G. (1996). Comorbidity and the general neurotic syndrome. British Journal of Psychiatry 168 (suppl. 30), 76-84.

Angst, J. \& Koch, R. (1991). Neurasthenia in young adults. In Problems of Psychiatry in General Practice (ed. M. Gastpar and P. Kielholz), pp. 37-48. Hogrefe \& Huber Publishers: Lewiston, NY.

Bates, D. W., Schmitt, W., Buchwald, D., Ware, N. C., Lee, J., Thoyer, E., Kornish, R. J. \& Komaroff, A. L. (1993). Prevalence of fatigue and chronic fatigue syndrome in a primary care practice. Archives of Internal Medicine 153, 2759-2765.

Bedford, A., Foulds, G. A. \& Sheffield, B. F. (1976). A new personal disturbance scale (DSSI/sAD). British Journal of Social and Clinical Psychology 15, 387-394.

Chen, M. (1986). The epidemiology of self-perceived fatigue among adults. Preventive Medicine 15, 74-81.

David, A., Pelosi, A., McDonald, E., Stephens, D., Ledger, D., Rathbone, R. \& Mann, A. (1990). Tired, weak, or in need of rest: fatigue among general practice attenders. British Medical Journal 301, 1199-1202.

Duffy, D. L. (1994). Asthma and Allergic Diseases in Australian Twins and their Families. Ph.D. thesis. University of Queensland: Brisbane, Australia.

Fukuda, K., Straus, S. E., Hickie, I., Sharpe, M. C., Dobbins, J. G. \& Komaroff, A. (1994). The chronic fatigue syndrome: a comprehensive approach to its definition and study. Annals of Internal Medicine 121, 953-959.

Goldberg, D. (1996). A dimensional model for common mental disorders. British Journal of Psychiatry 168 (suppl. 30), 44-49.

Goldberg, D. \& Bridges, K. (1991). Minor psychiatric disorders and neurasthenia in general practice. In Problems of Psychiatry in General Practice (ed. M. Gastpar and P. Kielholz), pp. 79-88. Hogrefe \& Huber: Lewiston, NY.

Goldberg, D. \& Williams, P. (1988). A User's Guide to the General Health Questionnaire. NFER-Nelson: Windsor.
Goldberg, D. P., Bridges, K., Duncan-Jones, P. \& Grayson, D. (1987). Dimensions of neuroses seen in primary care settings. Psychological Medicine 17, 461-470.

Goodchild, M. E. \& Duncan-Jones, P. (1985). Chronicity and the General Health Questionnaire. British Journal of Psychiatry 146, 55-61.

Hickie, I., Lloyd, A., Wakefield, D. \& Parker, G. (1990). The psychiatric status of patients with chronic fatigue syndrome. British Journal of Psychiatry 156, 534-540.

Hickie, I., Lloyd, A., Hadzi-Pavlovic, D., Parker, G., Bird, K. \& Wakefield, D. (1995). Can the chronic fatigue syndrome be defined by distinct clinical features? Psychological Medicine 25, 925-935.

Hickie, I., Hooker, A. W., Hadzi-Pavlovic, D., Bennett, B. K., Wilson, A. J. \& Lloyd, A. R. (1996). Fatigue in selected primary care settings: sociodemographic and psychiatric correlates. Medical Journal of Australia 164, 585-588.

Hickie, I., Hadzi-Pavlovic, D. \& Ricci, C. (1997). Reviving the diagnosis of neurasthenia. Psychological Medicine 27, 989-994.

Hickie, I., Koschera, A., Hadzi-Pavlovic, D., Bennett, B., Lloyd, A. $(1998 a)$. The temporal stability and co-morbidity of prolonged fatigue: a longitudinal study in primary care. (Submitted.)

Hickie, I., Scot, E. M. \& Davenport, T. A. (1998b). Somatic distress: developing more integrated concepts. Current Opinion in Psychiatry 11, 153-158.

Hickie, I., Bennett, B., Lloyd, A., Heath, A. \& Martin, N. (1999). Complex genetic and environmental relationships between psychological distress, fatigue and immune functioning: a twin study. Psychological Medicine 29, 269-277.

Hunt, C. \& Andrews, G. (1995). Comorbidity of anxiety disorders: life chart approach. Journal of Psychiatric Research 29, 467-480.

Joreskog, K. G. \& Sorbom, D. (1993). New features in PRELIS 2 and LISREL 8. Scientific Software Inc.: Chicago.

Kendell, R. E. (1989). Clinical validity. Psychological Medicine 19, 45-55.

Kendler, K. S. (1996). Major depression and generalised anxiety disorder. Same genes, (partly) different environments - revisited. British Journal of Psychiatry 30, 68-75.

Kendler, K. S. \& Karkowski-Shuman, L. (1997). Stressful life events and genetic liability to major depression: genetic control of exposure to the environment? Psychological Medicine 27, 539-547.

Kendler, K., Heath, A., Martin, N. \& Eaves, L. J. (1986). Symptoms of anxiety and depression in a volunteer twin population. The etiologic role of genetic and environmental factors. Archives of General Psychiatry 43, 213-221.

Kendler, K. S., Heath, A. C., Martin, N. G. \& Eaves, L. J. (1987). Symptoms of anxiety and symptoms of depression. Same genes, different environments? Archives of General Psychiatry 44, 451457.

Kendler, K. S., Neale, M. C., Kessler, R. C., Heath, A. C. \& Eaves, L. J. (1992). Major depression and generalized anxiety disorder. Same genes, (partly) different environments? Archives of General Psychiatry 49, 716-722.

Kessler, R. C., McGonagle, K. A., Zhao, S., Nelson, C. B., Hughes, M., Eshleman, S., Wittchen, H. \& Kendler, K. S. (1994). Lifetime and 12-month prevalence of DSM-III-R psychiatric disorders in the United States: results from the National Comorbidity Survey. Archives of General Psychiatry 51, 8-19.

Kirmayer, L. J. \& Robbins, J. M. (1991). Functional somatic syndromes. In Current Concepts of Somatization: Research and Clinical Perspectives (ed. L. J. Kirmayer and J. M. Robbins), pp. 79-106. American Psychiatric Press: Washington, DC.

Kroenke, K., Wood, D. R., Mangelsdorff, D., Meier, N. J. \& Powell, J. B. (1988). Chronic fatigue in primary care. Journal of the American Medical Association 260, 929-934.

Martin, N. G. \& Martin, P. G. (1975). The inheritance of scholastic abilities in a sample of twins. I. Ascertainments of the sample and diagnosis of zygosity. Annals of Human Genetics 39, 213-218.

Mason, P. \& Wilkinson, G. (1996). The prevalence of psychiatric morbidity OPCS : survey of psychiatric morbidity in Great Britain. British Journal of Psychiatry 168, 1-3.

Merikangas, K. \& Angst, J. (1994). Neurasthenia in a longitudinal 
cohort study of young adults. Psychological Medicine 24, 10131024.

Neale, M. C. \& Cardon, L. R. (1992). Methodology for Genetic Studies of Twins and Families. Kluwer Academic Publishers: Dordrecht.

Neale, M. C. \& Eaves, L. J. (1993). Estimating and controlling for the effects of volunteer bias with pairs of relatives. Behavior Genetics 23, 271-277.

Ooki, S., Yamada, K., Asaka, A. \& Hayakawa, K. (1990). Zygosity diagnosis of twins by questionnaire. Acta Geneticae Medicae et Gemellologiae (Roma) 39, 109-115.

Pawlikowska, T., Chalder, T., Hirsch, S. R., Wallace, P., Wright, D. J. M. \& Wessley, S. C. (1994). Population based study of fatigue and psychological distress. British Medical Journal 308, $763-766$.

Roy, M. A., Neale, M. C., Pedersen, N. L., Mathe, A. A. \& Kendler, K. S. (1995). A twin study of generalized anxiety disorder and major depression. Psychological Medicine 25, 1037-1049.

Üstün, T. B. \& Sartorius, N. (eds.) (1995). Mental Illness in General Health Care: An International Study. John Wiley \& Sons: Chichester.

Vercoulen, J. H. H. M., Swanink, C. M. A., Zitman, F. G., Vreden,
S. G. S., Hoofs, M. P. E., Fennis, J. F. M., Galama, J. M. D., van der Meer, J. W. M. \& Bleijenberg, G. (1996). Randomised, doubleblind, placebo-controlled study of fluoxetine in chronic fatigue syndrome. Lancet 347, 858-861.

Wessely, S. (1990). Old wine in new bottles: neurasthenia and 'ME'. Psychological Medicine 20, 35-53.

Wessely, S. \& Powell, R. (1989). Fatigue syndromes: a comparison of chronic postviral fatigue with neuromuscular and affective disorders. Journal of Neurology, Neurosurgery and Psychiatry 52, 940-948.

Wessely, S., Chalder, T., Hirsch, S., Wallace, P. \& Wright, D. (1996). Psychological symptoms, somatic symptoms, and psychiatric disorder in chronic fatigue and chronic fatigue syndrome: a prospective study in the primary care setting. American Journal of Psychiatry 153, 1050-1059.

Wilson, A., Hickie, I., Wright, M., Bennett, B. \& Wakefield, D. (1994). Moclobemide in chronic fatigue syndrome: a double blind, placebo-controlled trial. Neuropsychopharmacology 10 (No. 3S), 245.

World Health Organization (1992). The Tenth Revision of the International Classification of Diseases and Related Health Problems (ICD-10). WHO: Geneva. 